\title{
Feature study of hysterical blindness EEG based on FastICA with combined-channel information
}

\author{
Xuying Qin, Wei Wang*, Lintao Hu, Xu Wang and Xiaojie Yuan \\ Institute of Biomedical Engineering, School of Science and Information, Lanzhou University, Lanzhou, \\ Gansu, China
}

\begin{abstract}
.
BACKGROUND: An appropriate feature study of hysteria electroencephalograms (EEG) would provide new insights into neural mechanisms of the disease, and also make improvements in patient diagnosis and management.

OBJECTIVE: The objective of this paper is to provide an explanation for what causes a particular visual loss, by associating the features of hysterical blindness EEG with brain function.

METHODS: An idea for the novel feature extraction for hysterical blindness EEG, utilizing combined-channel information, was applied in this paper. After channels had been combined, the sliding-window-FastICA was applied to process the combined normal EEG and hysteria EEG, respectively. Kurtosis features were calculated from the processed signals. As the comparison feature, the power spectral density of normal and hysteria EEG were computed.

RESULTS: According to the feature analysis results, a region of brain dysfunction was located at the occipital lobe, O1 and $\mathrm{O} 2$. Furthermore, new abnormality was found at the parietal lobe, $\mathrm{C} 3, \mathrm{C} 4, \mathrm{P} 3$, and $\mathrm{P} 4$, that provided us with a new perspective for understanding hysterical blindness.

CONCLUSIONS: Indicated by the kurtosis results which were consistent with brain function and the clinical diagnosis, our method was found to be a useful tool to capture features in hysterical blindness EEG.
\end{abstract}

Keywords: Hysterical blindness EEG, combined-channel, kurtosis feature, FastICA

\section{Introduction}

Hysteria, a formerly common expression, refers to a type of disease without a recognized organic illness. Previously, hysteria was exclusively connected with women, but during World War I some related, classical symptoms were also found in men, including blindness, deafness, and so on [1]. Hysteria may also involve other complex and varied clinical symptoms. They can be roughly divided into several main conditions, between which there is considerable overlap: a. Conversion disorder, b. Somatization disorder, c. Hypochondriasis, d. Pain disorder, e. Factitious disorder, and other disorders affected by psychological factors [2]. Patients with conversion disorders may present symptoms such as convulsions, hearing loss, and visual loss displaying as weak sight or blindness.

\footnotetext{
${ }^{*}$ Corresponding author: Wei Wang, Institute of Biomedical Engineering, School of Science and Information, Lanzhou University, South Tianshui Road 222, Lanzhou 730000, Gansu, China. Tel.: +86 1391925 3607; Fax: +86 0931891 2778; E-mail: wangw@lzu.edu.cn.
} 
Patients with hysteria are more likely to cause harm and damage or commit crimes. In certain cases, a healthy person may intentionally pretend to have some hysterical symptoms. However, current identification of hysteria still largely depends on clinical experience. There are still relatively few studies on hysteria EEG feature as well as relationship analysis between hysteria and brain function. So, the related study of hysteria EEG may not only be beneficial to the correct diagnosis and treatment, but may also provide significant evidence for discerning whether or not a person is feigning hysteria.

Existing studies of hysteria diagnosis using modern techniques include the hysteria provocative test by EEG (EEG-HPT) [3], video EEG (VEEG) [4], brain electrical activity mapping (BEAM) [5], evoked potentials [6], functional magnetic resonance imaging(FMRI) [7], positron emission tomography(PET) [8], magnetoencephalography (MEG) [9], and so on. Thomas Willis first pointed out hysteria was caused by brain disease [10]. However, the underlying brain mechanisms tend to be poorly understood. Hysteria EEG of different symptoms are generally near normal, although EEG of the typical hysteria convulsions can be slightly abnormal [11], and nonspecific EEG abnormalities often appear in hysterical seizures that are caused by organic diseases [12]. However, some recent brain function imaging studies of hysteria have revealed that different parts of brain regions displayed activity changes when the patients were attacked by hysterical disorders, such as decreased activity in the visual cortex during hysterical blindness [13]. The EEG of hysterical visual loss was studied in this paper. The diagnosis was given to be simply a mild abnormality before our experiment was finished.

Based on biological sense, the combined-channel, a new idea for the feature extraction of hysterical blindness EEG, was adopted in this paper. After channels had been combined, FastICA was used to extract normal and hysteria kurtosis features in the way of sliding window. At the same time, the power spectral density (PSD) of each normal and hysteria channel was computed for comparison with kurtosis. According to kurtosis feature results, the abnormal channels were probed, and relationships to brain function areas were analyzed by associating the distribution of different brain dysfunction regions.

\section{Methods and theories}

\subsection{Independent Component Analysis}

Independent Component Analysis (ICA), an expansion of Principal Component Analysis (PCA), is a recently developed method for analyzing data and signals. Essentially, ICA is a method to extract independent components from the observed signals which was first proposed as the blind source separation (BSS) of linear mixed signals [14]. The basic model is as follows:

$m$ independent signals $x_{1}(k), x_{2}(k) \ldots x_{m}(k)$ were linearly mixed to form $y_{1}(k), y_{2}(k) \ldots y_{n}(k)$, where $k$ denotes time. If time delay and other interference factors are ignored, the mixing process can be expressed as:

$$
y_{i}(k)=a_{i 1} x_{1}(k)+a_{i 2} x_{2}(k)+\ldots+a_{i m} x_{m}(k)
$$

where $a_{i j}(i=1,2 \ldots n ; j=1,2 \ldots m ; n \geqslant m)$ are the weight coefficients. $x_{j}(k)(j=1 \ldots m)$ can be recovered from the mixed signals $y_{i}(k)(i=1 \ldots n)$ only by ICA.

A simple representation becomes the formula (2)

$$
Y=A X
$$


with the matrix $A$ denoting $a_{i j}$, vector $X$ referring to the source signals, and vector $Y$ standing for the mixed signals.

Therefore, if $W$ is the inverse of $A$, the independent sources $X$ can be obtained through the calculation Eq. (3):

$$
X=W Y
$$

As EEG basically satisfies the assumptions of ICA: a. Different current sources are mixed at the electrodes, b. Time delay can be neglected during the transmission of the current signal, c. Number of sources is equal to or fewer than the number of mixed signals, and d. Each independent source is non-Gaussian [15].

\subsection{Fast fixed-point algorithm for Independent Component Analysis}

The fast fixed-point algorithm for ICA (FastICA) [16] was applied to process the EEG signals for its fast convergence and other own advantages compared with other algorithms.

To realize FastICA, four steps are necessary. 1). Randomly construct an initial vector $w(j)$ with unit norm, and $j=1$ at first. 2). Calculate $w(j+1)=E\left\{Y\left[w(j)^{T} Y\right]^{3}\right\}-3 w(j)$, where $E$ means computing the expectation. 3). Make $w(j)$ dived by its norm. 4). See whether $\left|w(j+1)^{T} w(j)\right|$ is very close to 1 . if not, let $j=j+1$ and jump to step 2; otherwise output $w(j)$. Vector $w(j)(j=1 \ldots m)$ is just one column of the orthogonal mixing matrix which corresponds to one independent signal. To extract all the $m$ components, the algorithm should be run $m$ times.

ICA is usually used to process a large segment of signals, and consequently, weak changes and detailed information are ignored. However, the Sliding-window-FastICA can remedy this problem because new points would come into the sliding window for every slide: $0-W, L-W+L, 2 L-W+2 L \ldots$, where $W$ is the window length, and $L$ represents the sliding step length. Furthermore, the sliding method has been proven to detect robust activation results by some research teams [17]. Additionally, it makes the input of FastICA use fewer points, thus reducing the computation.

\subsection{Kurtosis}

Kurtosis, a dimensionless parameter, is usually used to reflect the distribution characteristics of a vibration signal. The relevant applications [18] can indicate the non-stationarity and detect the transient components of a noisy signal. It is defined as Eq. (4), where $u$ denotes the centered random variable, and $E$ means calculating the expectation. Since $u$ has unit variance, the simplification is as the expression Eq. (5).

$$
\begin{aligned}
& \operatorname{kurt}(u)=E\left\{u^{4}\right\}-3\left(E\left\{u^{2}\right\}\right)^{2} \\
& \operatorname{kurt}(u)=E\left\{u^{4}\right\}-3
\end{aligned}
$$

In this paper, the normalized kurtosis was computed as formula (6) by the discrete random variable $z$ with $\mu$ for the mean of $u, \sigma$ for the variance of $u$, and $E$ for getting the expectation.

$$
k=\frac{E(z-\mu)^{4}}{\sigma^{4}}
$$




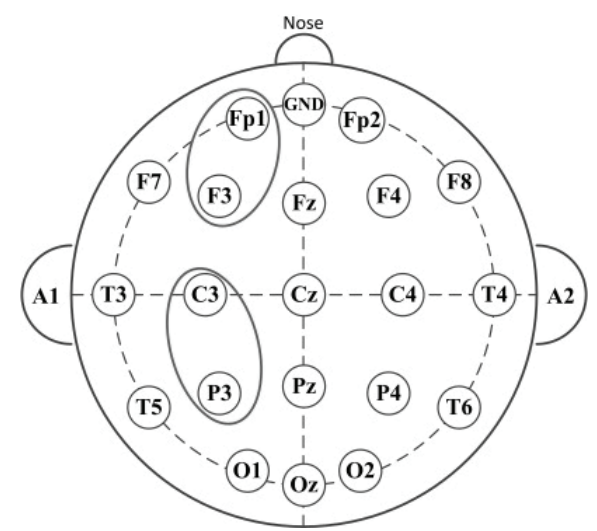

Fig. 1. The international 10-20 electrode placement system. Each two electrodes in a circle represent an example of a combined-channel of adjacent electrodes.

\subsection{Power spectral density}

PSD can characterize how the power of signals distributes along frequencies. It is a classical method for EEG signal processing that can interpret amplitude changes of EEG in the time domain into power variation in the frequency domain. As PSD is only suitable for wide-sense stationary signal, EEG is assumed to be quasi-stationary. For signal $x(t)$ in a finite interval $[0, T]$, its Fourier transform is given by:

$$
X(f)=\int_{0}^{T} x(t) e^{-i 2 \pi f t} d t
$$

Thus, the PSD can be computed by:

$$
S_{x}(f)=\lim _{T \rightarrow \infty} \frac{1}{T}|X(f)|^{2}
$$

\section{Feature extraction}

The studied EEG signals were separately recorded from one normal person and one hysteria patient in People's Hospital of Gansu Province. The EEG was recorded according to the international 10-20 electrode placement, as shown in Fig. 1, with a sample rate of $200 \mathrm{~Hz}$. In this paper, $5 \mathrm{~s}$ duration of every 16 channels in both normal EEG and hysteria EEG were chosen to be analyzed. The normal EEG of channels F7 and F8, as well as the hysteria EEG of channel F7, F8, P3, P4, O1, O2, T5, and T6, were shown in Fig. 2. Through observing the original normal and hysteria EEG signals, the amplitude and fluctuations of each normal channel were smaller than the corresponding hysteria channel. It was strongly indicated in hysteria channels $\mathrm{P} 3, \mathrm{P} 4, \mathrm{O} 1$, and $\mathrm{O} 2$, which held obviously higher amplitudes as compared to normal F7 and F8 channels and other hysteria channels. But the amplitude of hysteria EEG was in a small range of $[-50,50] \mu \mathrm{V}$. It was one fundamental characteristic of hysteria EEG that differs from the epileptic EEG which exhibits sharp waves or spike waves with high amplitude. In clinics, diagnosis of hysteria primarily refers to the behavior and description of the patients themselves. Otherwise hysteria could not be precisely diagnosed simply by visual inspection of hysteria EEG, let alone the abnormalities hidden 

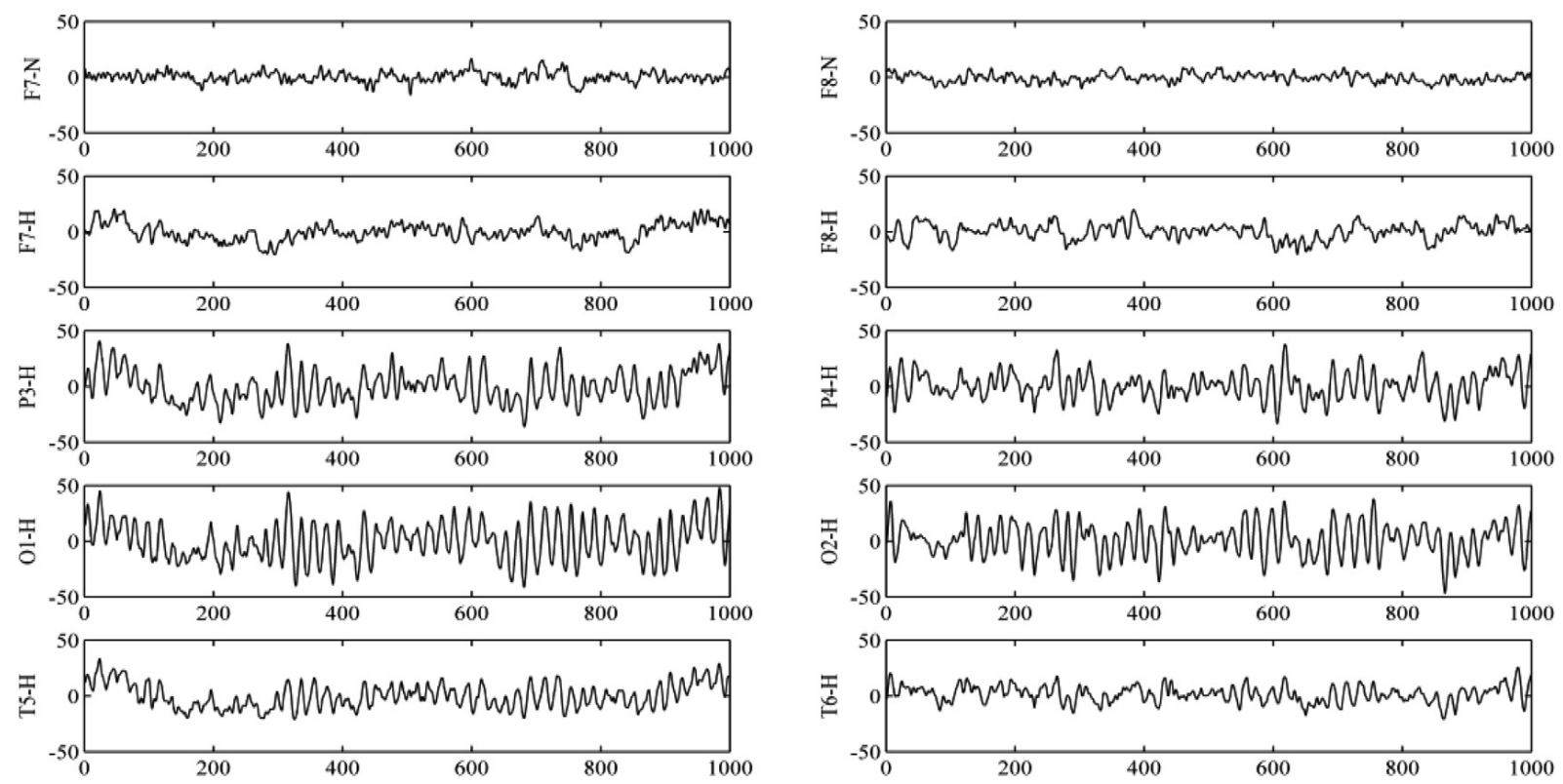

Fig. 2. Comparison of the original normal and hysteria EEG (AD = $200 \mathrm{~Hz})$. Hysteria EEG: channel F7, F8, P3, P4, O1, O2, T5, and T6 that are marked by H. Normal EEG: channel F7 and F8 that are marked by $N$.

within the hysteria EEG. Additionally, for normal EEG, signals from each two channels with symmetrical distribution of electrodes on the brain performed similar waveforms. By contrast, it was relatively worse for hysteria channel pairs $\mathrm{O} 1$ and $\mathrm{O} 2, \mathrm{~T} 5$ and T6 with regard to the symmetry. This fact suggested these abnormal channels are closely related to the brain dysfunction regions.

\subsection{Kurtosis feature}

Each pair of adjacent channels (the example pairs Fp1-F3 and C3-P3 in Fig. 1, the same as other channels) and each pair of symmetrical channels (such as Fp1-Fp2 and F7-F8) were combined under normal and hysteria states. Each signal in a combined-channel was perceived as the reference of each other. For convenience, the combined-channels were separately named as adjacent combined-channel (A-CC) and symmetrical combined-channel (S-CC).

The sliding-window-FastICA was applied to process the combined-channel EEG. The optimal window length $W$ was set at 200, and the step size $L$ was set at 20. The sliding window was made to traverse the entire signal by sliding the setting step size at a time: $0-200,20-220,40-240, \ldots, 800-1000$. In the procedure, there were 41 sliding windows in total. Every time, after being processed by one sliding window, the kurtosis of each channel was computed. Finally, the median of the whole kurtosis value $(N=$ 41) corresponding to one channel was taken as its feature.

There were 30 groups of A-CC altogether. Since the entire feature data was too much, only 8 groups having significant difference of hysteria EEG compared with the normal EEG were shown in Table 1. Every hysteria feature was larger than the normal feature of the same channel. It was especially representative for the features of F4-C4, C3-F7, C4-F8, P3-O1, P3-T3, P4-T4, O1-T5, and O2-T6. In Table 1, all the features of normal EEG in channel 1 or channel 2 column were treated as a group of normal feature set, the same as the features of hysteria EEG. $T$-test between the normal and hysteria features 
Table 1

Kurtosis features of adjacent combined-channels under normal and hysteria state $(N=41)$. In each combined channel, the former represented channel 1, and the latter represented channel 2. All kurtosis features of normal or hysteria EEG in the Channel 1 column are regarded as one normal or hysteria feature set, the same as Channel 2

\begin{tabular}{lccccc}
\hline Combined-channel & \multicolumn{2}{c}{ Normal } & & \multicolumn{2}{c}{ Hysteria } \\
\cline { 2 - 3 } \cline { 5 - 6 } Channel 1-Channel 2 & Channel 1 & Channel 2 & & Channel 1 & Channel 2 \\
\hline F4-C4 & $2.332 \pm 0.192$ & $2.712 \pm 0.179$ & & $2.548 \pm 0.529$ & $2.961 \pm 0.255$ \\
C3-F7 & $2.344 \pm 0.197$ & $2.733 \pm 0.228$ & & $2.598 \pm 0.524$ & $3.048 \pm 0.411$ \\
C4-F8 & $2.327 \pm 0.226$ & $2.728 \pm 0.156$ & & $2.544 \pm 0.425$ & $3.093 \pm 0.361$ \\
P3-O1 & $2.185 \pm 0.205$ & $2.815 \pm 0.343$ & & $2.538 \pm 0.498$ & $2.904 \pm 0.379$ \\
P3-T3 & $2.357 \pm 0.148$ & $2.771 \pm 0.177$ & & $2.543 \pm 0.518$ & $2.951 \pm 0.450$ \\
P4-T4 & $2.320 \pm 0.180$ & $2.733 \pm 0.197$ & & $2.530 \pm 0.309$ & $3.074 \pm 0.321$ \\
O1-T5 & $2.353 \pm 0.214$ & $2.703 \pm 0.203$ & & $2.546 \pm 0.518$ & $3.046 \pm 0.383$ \\
O2-T6 & $2.339 \pm 0.184$ & $2.746 \pm 0.171$ & & $2.525 \pm 0.321$ & $3.040 \pm 0.352$ \\
\hline
\end{tabular}

Table 2

Kurtosis features of symmetrical combined-channels under normal and hysteria state $(N=41)$. All kurtosis features of normal or hysteria EEG in the Channel 1 column are regarded as one normal or hysteria feature set, the same as Channel 2

\begin{tabular}{lccccc}
\hline Combined-channel & \multicolumn{2}{c}{ Normal } & & \multicolumn{2}{c}{ Hysteria } \\
\cline { 2 - 3 } \cline { 5 - 6 } Channel 1-Chanel 2 & Channel 1 & Channel 2 & & Channel 1 & Channel 2 \\
\hline Fp1-Fp2 & $2.332 \pm 0.222$ & $2.677 \pm 0.256$ & & $2.559 \pm 0.190$ & \\
F7-F8 & $2.326 \pm 0.162$ & $2.714 \pm 0.308$ & & $2.562 \pm 0.210$ & 0.086 \\
F3-F4 & $2.433 \pm 0.181$ & $2.634 \pm 0.255$ & & $2.585 \pm 0.276$ & $2.830 \pm 0.154$ \\
C3-C4 & $2.407 \pm 0.187$ & $2.714 \pm 0.364$ & & $2.585 \pm 0.179$ & \\
T3-T4 & $2.300 \pm 0.177$ & $2.714 \pm 0.271$ & & $2.446 \pm 1.006$ & $2.871 \pm 0.153$ \\
P3-P4 & $2.408 \pm 0.217$ & $2.600 \pm 0.231$ & & $2.585 \pm 0.216$ & $2.822 \pm 0.330$ \\
T5-T6 & $2.332 \pm 0.152$ & $2.768 \pm 0.551$ & & $2.665 \pm 0.190$ & $2.772 \pm 0.160$ \\
O1-O2 & $2.332 \pm 0.180$ & $2.724 \pm 0.501$ & & $2.584 \pm 0.213$ & $2.909 \pm 0.137$ \\
\hline
\end{tabular}

Table 3

PSD distribution of 16 channels EEG under normal and hysteria state $(f<30 \mathrm{~Hz})$. All features of normal EEG in the Normal row are regarded as one normal feature set, the same as the hysteria feature set

\begin{tabular}{lllllllll}
\hline Channel & Fp1 & Fp2 & F3 & F4 & C3 & C4 & P3 & P4 \\
Normal & 257.161 & 228.915 & 220.120 & 200.355 & 194.860 & 232.207 & 159.179 & 263.254 \\
Hysteria & 681.396 & 598.432 & 562.877 & 498.795 & 670.117 & 487.671 & 920.209 & 726.092 \\
\hline Channel & O1 & O2 & F7 & F8 & T3 & T4 & T5 & T6 \\
Normal & 122.610 & 96.407 & 126.943 & 72.714 & 158.331 & 95.349 & 95.774 & 57.451 \\
Hysteria & 1385 & 1164.3 & 194.372 & 259.304 & 246.930 & 277.359 & 417.331 & 241.321 \\
\hline
\end{tabular}

were channel 1: $p<4.106 \mathrm{E}-06$ and channel 2: $p<4$.177E-06. For each listed A-CC, the kurtosis characteristics of channel 1 were all more than 2.5, and the kurtosis features of channel 2 were all more than 2.9. These channels were preliminary identified to be abnormal.

In terms of S-CC features, it was evident that normal features were smaller than hysteria features in the same channel. Under the same hypothesis of normal and hysteria feature set as A-CC, the $t$-test between normal and hysteria features were channel 1: $p<1.435 \mathrm{E}-05$, channel 2: $p<0.00028$. The much higher amplitude appeared in $\mathrm{O} 1-\mathrm{O} 2$ under hysteria state, where the kurtosis of channel 1 was over 2.5 and the kurtosis of channel 2 was over 2.9. Although C3-C4, F3-F4, and P3-P4 did not present the same large hysteria features as $\mathrm{O} 1-\mathrm{O} 2$, they still held greater values of both channels relative to other S-CCs. Therefore, $\mathrm{O} 1, \mathrm{O} 2, \mathrm{P} 3, \mathrm{P} 4, \mathrm{C} 3$, and $\mathrm{C} 4$ were classified as abnormal channels because both their A-CC features and S-CC features showed unusual large value. 

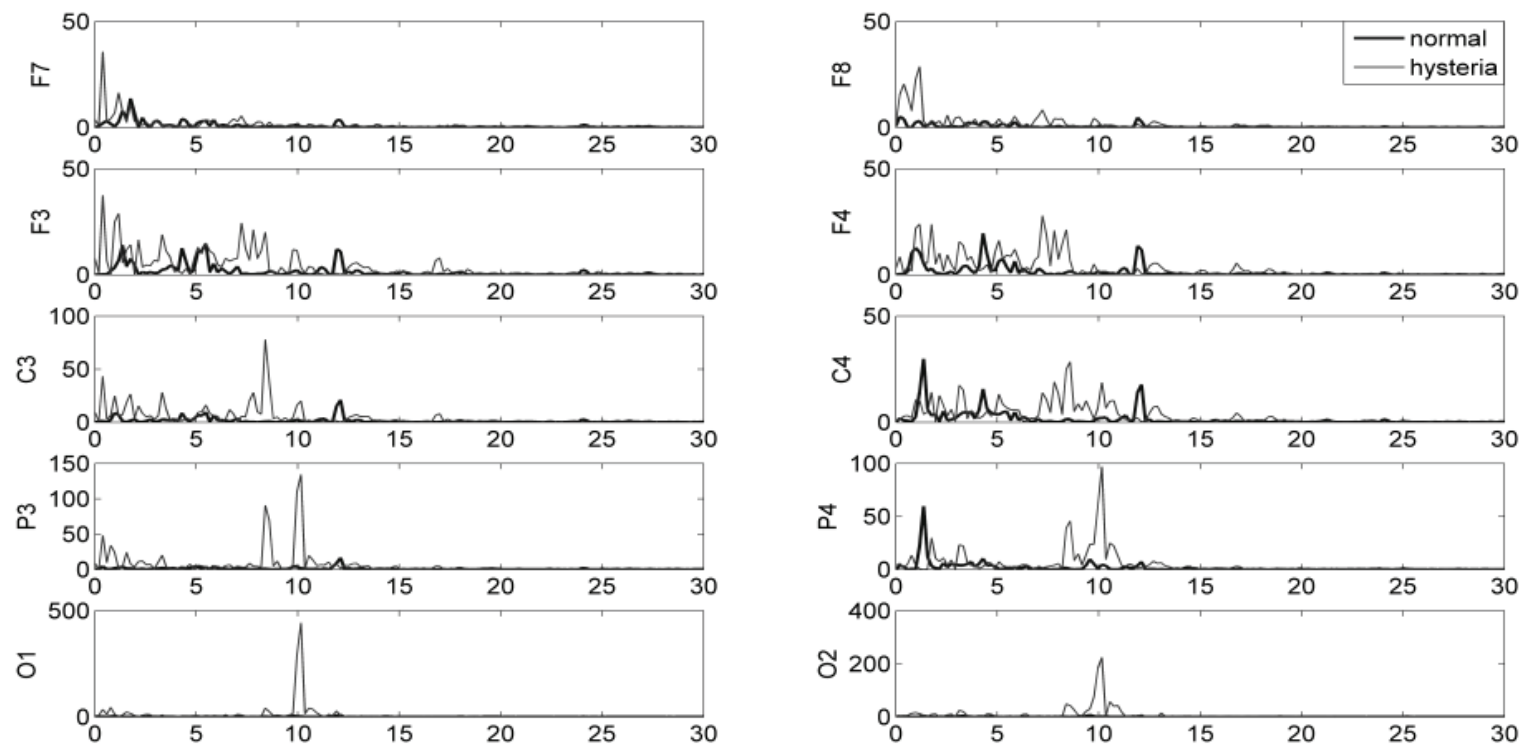

Fig. 3. PSD of normal and hysteria EEG of some channels in the range of $30 \mathrm{~Hz}$. The thick line illustrates the PSD of a normal EEG. The thin line illustrates the PSD of a hysteria EEG.

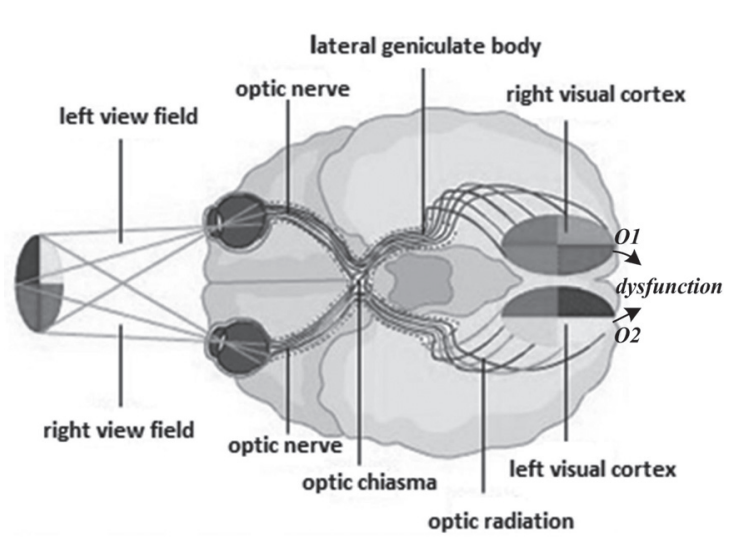

Fig. 4. Optic nerve pathways [21].
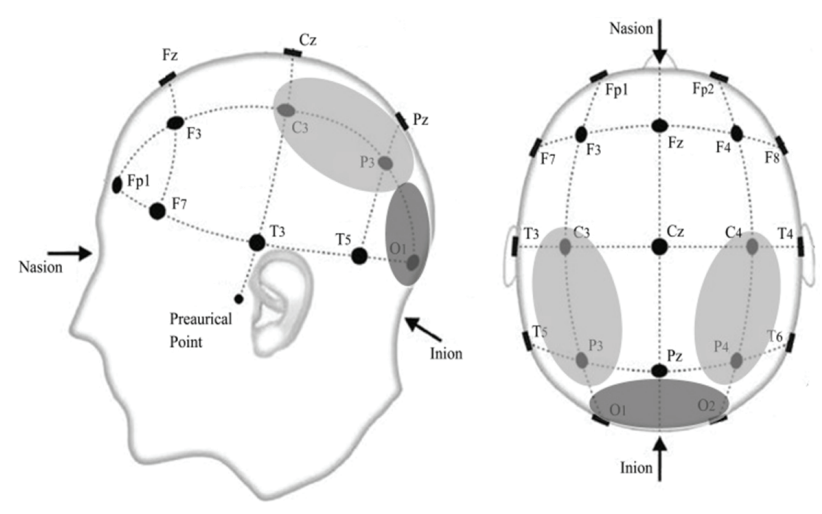

Fig. 5. Localization of abnormal brain areas associated with hysterical blindness. The dark color area is the dysfunction occipital lobe. The light color areas indicate the abnormal parietal lobe.

\subsection{Power spectral density feature}

To compare the performance with the kurtosis feature results, the PSD distribution of each single channel under normal and hysteria states was computed. Before the estimation, the 5-second signals were zero padded to 1024 points. As demonstrated by the thick line for the PSD of normal EEG and the thin line for the PSD of hysteria EEG in Fig. 3, it was evident that the amplitude of each normal PSD was within a small range, whereas the hysteria PSD of some channels had abnormally high values. For example, the hysteria PSD of channel O1, O2, and P3 at $10.16 \mathrm{~Hz}$ were separately 443.577, 224.098, and 134.434.

As the PSD of normal and hysteria EEG in each channel were concentrated in a small frequency range, the power distributions below $30 \mathrm{~Hz}$ were calculated and shown in Table 3. The hysteria PSD was 
distinctly greater. The 16 PSD values of normal EEG were regarded as the normal feature set, the same as the hysteria feature set. Thus, t-test between normal and hysteria feature set was $p<7.99 \mathrm{E}-05$. The power of $\mathrm{O} 1, \mathrm{O} 2, \mathrm{P} 3$, and $\mathrm{P} 4$ were quite larger relative to all normal channels and other hysteria channels. In other words, abnormalities appeared in these channels, which was in accordance with the associated conclusion from the overall kurtosis features and also consistent with the original EEG waveforms. But PSD did not detect anything obviously abnormal in $\mathrm{C} 3$ and $\mathrm{C} 4$, whereas the kurtosis feature did detect.

\section{Conclusion and discussion}

The original hysteria EEG in this paper seemed to be mildly abnormal. After the kurtosis features of hysteria and normal EEG had been analyzed, the sites of electrodes with evidently large features in both A-CC and S-CC were regarded as abnormal brain regions. According to the experimental results in Tables 1 and 2, the brain dysfunction area was located at the occipital lobe where electrodes O1 and $\mathrm{O} 2$ were placed. As the comparison feature, the PSD of $\mathrm{O} 1$ and $\mathrm{O} 2$ also presented great value, which performed consistency with the kurtosis features about the distribution of feature values in all channels. Hysterical blindness was caused by the visual cortex inhibition which was often non-uniform and insufficient [19]. From the optic nerve pathways demonstrated in Fig. 4, it was clear that when the optic nerve was normal, the visual cortex decided whether or not eyes could see. Thus, the conclusion that the brain dysfunction region was the occipital lobe with electrodes $\mathrm{O} 1$ and $\mathrm{O} 2$ agreed with visual brain function. Our conclusion remained consistent with the clinical diagnosis of hysterical blindness. It meant changes did exist in brain activity at the visual cortex during hysterical blindness.

Furthermore, a new abnormal area was located at the parietal lobe with P3, P4, C3, and C4 through our method. This new discovery suggests that hysteria blindness with dysfunction at the visual cortex concerned the parietal lobe, which might be attributable to the role of the parietal cortex in brain function. Activity of the parietal region was associated with the brain's other lobes and could assist the occipital lobe for visual judgments. And some researches had demonstrated that superior parietal lobe was functionally specialized for spatial vision [20]. Therefore, in the studied case, if the occipital lobe displayed dysfunction, an abnormality would also appear at the closely function-related regions. The abnormalities were located in Fig. 5 with dark color area for the dysfunction occipital lobe, and light color areas for the abnormal parietal lobe. This result was confirmed to be accurate according to the established visual brain function theory. It provided a new enlightenment to discover a neural mechanism explaining what the particular hysterical visual loss was caused by. Further, it could offer a reference for comprehensive analysis of brain function changes related to hysterical blindness.

Hysterical blindness is quite common in ophthalmic diagnosis. It is fairly easy to be misdiagnosed as optic neuritis, which often causes delays in treatment. From the perspective of medical costs for the patients, such disease often causes a substantial burden. So, our study is of vital practical significance for accurate diagnosis and treatment. Also, it can provide scientific evidence to exclude the possibility of a person malingering. The proposed combined-channel using ICA based on the biological sense has

never been utilized in other EEG feature studies. It provides a new insight into the mixing principles of EEG signals. Especially for the S-CC, if dysfunction appears at any region of the brain, signals from symmetrical channels may not exhibit highly similar waveforms because functions of the symmetrical parts on the brain will become unbalanced. Instead of nonlinear and complex algorithms, our method, using FastICA, tends to extract convictive features in a linear way. The sliding window helps reveal more detailed information and small EEG changes. Another application in respect to feature extraction is kurtosis, which is widely used to detect changes in vibration signals. In our experiment, kurtosis showed 
excellent performance for extracting features from EEG signals. From kurtosis feature results, a new abnormality that had never been found before was revealed by our study, while PSD did not discover. That meant PSD could not reveal the hidden abnormality in the case of relatively weak signals, but the kurtosis could. Finally, the relationship analysis between hysterical blindness and brain function was accomplished, giving an answer as to why anomalies arise from the visual and parietal cortex. It proved that our method was a useful exploratory tool for hysterical blindness feature extraction and analysis. Accordingly, on the basis of this study, more scientific data analyzing methods of features need to be considered. Developing improvements to the algorithm to extract features that are more discriminative between hysterical blindness and normal states is also an area of future study.

\section{References}

[1] Merskey H. The history of pain and hysteria. NeuroRehabilitation. 1997; 8(3): 157-162.

[2] Teasell RW, Shapiro AP. Diagnosis of conversion disorders in a rehabilitation setting. NeuroRehabilitation. 1997; 8(3): 163-174.

[3] Cohen RJ, Suter C. Hysterial seizures suggestion as a provocative EEG test. Annals of Neurology. 1982; 11(4): 391-395.

[4] Ramani SV, Quesney LF, Olson D, Gumnit RJ. Diagnosis of hysterical seizures in epileptic patients. American Journal of Psychiatry. 1980; 137(6): 705-709.

[5] Duffy FH. Topograhic display of evoked potentials: clinical applications of brain electrical activity mapping (BEAM). Annals of the New York Academy of Sciences. 1982; 388(1): 183-196.

[6] Schoenfeld MA, Hassa T, Hopf J, Eulitz C, Schmidt R. Neural correlates of hysterical blindness. Cerebral Cortex. 2011; 21: 2394-2398.

[7] Cojan Y, Waber L, Carruzzo A, Vuilleumier P. Motor inhibition in hysterical conversion paralysis. Neuroimage. 2009; 47(3): 1026-1037.

[8] Tart CT. States of consciousness and state-specific sciences. Science. 1972; 176(4040): 1203-1210.

[9] Hoechstetter K, Meinck HM, Henningsen P, Scherg M, Rupp A. Psychogenic sensory loss: magnetic source imaging reveals normal tactile evoked activity of the human primary and secondary somatosensory cortex. Neuroscience Letters. 2002; 323(2): 137-140.

[10] Boeker H. Hysteria. In: Binder MD, Hirokawa N, Windhorst U, editors. Encyclopedia of Neuroscience. Berlin: Springer. 2009; p. 1903-1908.

[11] Cao JY. Comparison and analysis of clinical and electroencephalographic characteristics between hysterical mental disorder and epileptic mental disorder. Journal of Modern Electrophysiology. 2004; 11(3): 135-136.

[12] Lelliott PT, Fenwick P. Cerebral pathology in pseudoseizures. Acta Neurologica Scandinavica. 1991; 83: 129-132.

[13] Vuilleumier P. Hysterical conversion and brain function. Progress in Brain Research. 2005; 150: 309-329.

[14] Jutten C, Herault J. Blind separation of sources part I: An adaptive algorithm based on neuromimetic architecture. Signal Processing. 1991; 24(1): 1-10.

[15] Jung TP, Makeig S, Lee TW, McKeown MJ, Brown G, Bell AJ, et al. Independent component analysis of biomedical signals. in: Proceedings of the 2nd International Workshop on Independent Component Analysis and Blind Signal Separation (ICA2000), 2000 June 19-22; Helsinki. Helsinki: Helsinki University of Technology. 2000; p. 15-26. Available from: research.ics.aalto.fi/events/ica2000/proceedings.

[16] Hyvärinen A, Oja E. A fast fixed-point algorithm for independent component analysis. Neural Computation. 1997; 9: $1483-1492$.

[17] Kiviniemi V, Vire T, Remes JJ, Elseoud AA, Starck T, Tervonen O, et al. A Sliding Time-Window ICA Reveals Spatial Variability of the default mode network in time. Brain Connectivity. 2011; 1(4): 339-347.

[18] Dwyer R. Detection of non-gaussian signals by frequency domain kurtosis estimation. In: Proceedings of the 1983 Acoustics, Speech and Signal Processing IEEE International Conference on ICASSP '83; 1983 Apr 14-16; Boston, Massachusetts, USA. New York: IEEE; 1983 [cited 2014 May 20]. p. 607-610. Available from: IEEE Xplore.

[19] Wang XY, Wang XM. Analysis of six cases of misdiagnosed hysterical blindness. Medical Information. 2011; 24(3): 1444.

[20] Haxby JV, Grady CL, Horwitz B, Ungerleider LG, Mishkin M, Carson RE, et al. Dissociation of object and spatial visual processing pathways in human extrastriate cortex. Proceedings of the National Academy of Sciences. 1991; 88(5): 1621-1625.

[21] [cited 2014 Apr 2]. Available from: http://www.zhibeifw.com/big5/fjgc/fjykx_list.php?id=7533. 